\title{
Paul Claudel et Jean Charlot en Georgie
}

\author{
Nina Hellerstein \\ University of Georgia
}

Il y a dix ans, j'ai découvert sur le campus de mon université, l'université de Georgie à Athens, la présence de plusieurs fresques créées par le peintre franco-américain Jean Charlot, qui avait été le collaborateur et ami de Paul Claudel. Le fondateur de l'école des Beaux-Arts à l'université de Georgie, Lamar Dodd, avait étudié avec Charlot à New York et l'avait invité à s'installer à Athènes pendant la guerre pour décorer plusieurs bâtiments universitaires, dont le bâtiment des Beaux-Arts et celui du Journalisme, devenu depuis celui de la faculté de Commerce (Charlot Murals 19). Claudel et Charlot se sont connus également à New York pendant l'ambassade américaine de Claudel, vers les années 1928-33 (Correspondance Claudel-Milhaud 112). Ainsi, deux mondes éloignés, celui du poète-ambassadeur français et celui d'une grande université publique dans le Sud américain, se sont rencontrés sur le plan de la création artistique, grâce à Charlot. Né à Paris en 1898, émigré d'abord au Mexique, installé ensuite aux Etats-Unis continentaux et enfin à Hawaï, Charlot a eu une carrière extraordinairement variée et ouverte aux cultures du monde entier (Thompson : http://libweb.hawaii.edu/libdept/charlotcoll/ J_Charlot/charlotthompson.html). Les œuvres qu'il a créées sur le campus athénien sont consacrées à des sujets monumentaux et allégoriques qui conviennent à la dimension publique de l'art mural, mais traités d'une manière singulièrement humaine, et originale. C'est cette alliance entre l'authenticité humaine et la vision monumentale qui a suscité l'intérêt de Claudel et qui a contribué à la profonde sympathie entre les deux créateurs.

Les peintures murales du bâtiment de la faculté de Commerce, antérieurement celle du Journalisme, à l'université de Georgie sont consacrées à célébrer la vocation du journalisme. 


\section{Nina Hellerstein}

D'un côté, Charlot a illustré une scène de l'histoire précolombienne du Mexique, intitulée "Cortez Lands in Mexico » et sous-titrée «Anno DMI 1519 : Emperor Montezuma's Scouts Cover America's First Scoop. Cortez in Mexico ». (Figures 1-3). De l'autre, il choisit de représenter une scène parallèle de l'histoire contemporaine : "Paratroopers Land in Sicily », qu'il explique ainsi dans son texte, "Anno DMI 1944. Press and Cameramen Flash On The Spot News. World War II ». (Figures 4-5). Comme l'explique Charlot, " The conquest of America, in its day a bloody affair, brought Christianity to the new world. Today, against another background of turmoil and carnage, America frees Europe out of Festung Europa » (Charlot Murals 134). Tandis que la scène de la guerre européenne est évidemment inspirée par l'actualité, celle de la conquête espagnole témoigne des racines permanentes de Charlot dans l'art dit "primitif ", son intérêt profond pour les images folkloriques de toutes les cultures, et surtout son inspiration dans l'art précolombien. L'on aura noté que malgré cette célébration des origines indigènes de la culture américaine, Charlot insiste sur l'importance de sa conversion au christianisme. La perspective religieuse personnelle de Charlot comprend à la fois une profonde sympathie pour toutes les formes du sacré, et un authentique dévouement à la religion catholique. C'est une des causes majeures du lien intellectuel et esthétique entre lui et Claudel.

Une autre raison importante de leur entente est la préoccupation des deux créateurs avec le concept de la composition comme base de la création picturale. L'on sait que ce concept est central dans toute l'esthétique de Claudel, et dans sa critique d'art, il lui attribue une importance fondamentale (Lioure 23-7). Ainsi, le texte principal qu'il a consacré aux œuvres de Charlot, écrit pour une exposition à New York en 1931, insiste sur l'importance pour l'art de Charlot d'une conception concrète et sculpturale de la composition.

Jean Charlot est un constructeur, un compositeur, [...] chez lui la sensation de la masse et du poids autour d'un invisible fil à plomb n'est jamais absente, [...] c'est ici 


\section{Paul Claudel et Jean Charlot en Georgie}

mouvement de terrain qui ressemble à un immense corps étendu derrière lequel on voit la mer bleue. Sur le côté se dresse une tête colossale à deux visages dont l'un est tourné vers la mer et l'autre vers le public. Elle est faite de pierres à demi ruinées où poussent des fougères et manguiers et un grand serpent est enlacé autour. (Colomb 1162)

Cette image fascinante exprime sous une forme complexe l'affrontement entre le monde païen condamné par Claudel et le salut chrétien auquel les deux créateurs adhèrent profondément. Comme le remarque le fils et commentateur de Charlot, John Charlot, cette image suggère également l'image du Mexique comme Terre-Mère portant une corne d'abondance remplie de convertis potentiels (John Charlot 71). Toutes les images du Livre témoignent de cette même intelligence profonde mise au service de la signification du texte. Un exemple particulièrement subtil se voit dans l'une des dernières images de l'œuvre, illustrant la scène 7 de la deuxième partie, «Au Paradis de l'Idée ». L'artiste reprend une image de la petite reine Isabelle entourée de ses amies, illustrant la scène 8 de la première partie, et la reproduit dans des tons d'argent et de blanc (figures 13-14). Comme dit L'Explicateur,

Mais, comme vous voyez, tout est devenu blanc! Cristal et argent! on dirait un paysage de givre ou une mantille de soie blanche. Les fruits, les fleurs, les feuilles, les branches, tout cela est d'argent, avec un fantôme de couleur qui survit, le sol même est comme poudré de sucre [...] Tout recommence de nouveau dans la lumière et l'explication; ne voyez-vous pas que tout est devenu pur et blanc et que tout est peint sur la lumière comme avec de la lumière condensée, [...] Tout se dessine comme sur la pure lumière intellectuelle et les personnages avant qu'ils montent au paradis de l'amour, vous les voyez qui s'amusent un moment dans ce paradis de l'idée. (1181-1182) 


\section{Nina Hellerstein}

Charlot réalise les suggestions visuelles offertes par le texte luimême en représentant la transfiguration de l'état terrestre et historique en une experience purifiée, aérienne et lumineuse, à travers la transformation des couleurs. Par le langage de la littéralité visuelle, il exprime la plus profonde spiritualité.

Ainsi, cette œuvre illustre bien la sympathie complexe entre les deux créateurs, sympathie mêlée d'admiration pour le poète catholique de la part de Charlot, et d'admiration par Claudel pour l'artiste archéologue et primitif, si doué pour rendre la force sauvage des croyances et des cultures paysannes. La complexité vient de l'hostilité de Claudel envers les croyances non-chrétiennes, tandis que Charlot se sent profondément solidaire de ces formes d'élan vers le sacré qu'il considère comme les antécédents de son propre christianisme. Il évoque ainsi sa réaction au traitement claudélien des dieux aztèques, "moi un peu choqué de voir mes dieux ancestraux maltraités " (John Charlot 69). Pourtant, la tension entre ces deux façons de vivre le christianisme et ses rapports avec les religions qui l'ont précédé contribue à la richesse de la collaboration entre Claudel et Charlot. Si le poète dit de son artiste collaborateur, «C'est un barbare », « c'est, à n'en pas douter, le plus énergique des compliments » (Labriolle 129-36). Cette qualité d'authenticité, alliée à la sophistication du métier et à la profondeur sincère, mais en même temps humaniste, de sa foi chrétienne, ont fait de Charlot un collaborateur privilégié de Claudel. Les œuvres qu'il nous a laissées sur le campus de l'université de Georgie réunissent toutes ces qualités et montrent combien, comme Claudel, il était un authentique citoyen du monde entier.

\section{Ouvrages cités}

Charlot, Jean. Picture Book. New York : John Becker, 1933.

The Book of Christopher Columbus. New Haven : Yale UP, 1930.

Charlot, John. "Jean Charlot as Paul Claudel's Ixtlilxochitl ", The Journal of Intercultural Studies, Nos. 17-18 (1990-1991), 64-74. [http://www.hawaii.edu/jcf/JohnCharlotOnJean/Ixtl.pdf]. 


\section{Paul Claudel et Jean Charlot en Georgie}

Claudel, Paul. Correspondance Paul Claudel-Darius Milhaud. Cahiers Paul Claudel III. Ed. J. Petit. Paris : Gallimard, 1961.

—. "Jean Charlot». In CEurres en prose. Ed. J. Petit et C. Galpérine. Paris : Gallimard, «La Pléiade », 1965. 296-298.

—. Le Liure de Christophe Colomb. In Théatre II. Ed. J. Madaule et J. Petit. Paris : Gallimard, "La Pléiade », 1965, 1129-1188.

—. "Quelques Réflexions sur la Peinture Cubiste ». Euvres en prose. 258-62.

- The Book of Christopher Columbus. New Haven : Yale UP, 1930.

Dodd, Lamar, Eugene Payor and Jean Charlot. Charlot Murals in Georgia. Athens : UGA Press, 1945.

Labriolle, Jacqueline de. Les «Christophe Colomb » de Paul Claudel. Paris : Klincksieck, 1972.

Lioure, Michel. "Claudel et la Critique d'Artt ». La Revue des Lettres Modernes, n ${ }^{\text {os }}$ 510-15, «Claudel et l'Art», ed. J. Petit, 1977, p. 7-34.

Morse, Peter. Jean Charlot's Prints : A Catalogue Raisonné. Honolulu : The UP of Hawai and the Jean Charlot Foundation, 1976.

Thompson, Karen. « Jean Charlot : Artist and Scholar ». [http://libweb. hawaii.edu/libdept/charlotcoll/J_Charlot/charlotthompson.html]. 


\section{Cœur à corps claudélien perçu dans le syn- crétisme des cultures : Le Poète et le Shamisen}

\section{Hélène Poiré}

Animé du "goût de l'étranger " ", Claudel revêt, subrepticement, la peau du Shamisen, cet instrument de musique japonais, et celle du poète, dans un dialogue tiré de L'oiseau noir dans le Soleil levant, 1929, rendu sous la forme d'une quelconque apologie de l'Orient et de l'Occident.

Fébrile, l'ambassadeur poète se livre à un tour d'horizon élargi à travers des élucubrations qui ont tôt fait de l'inscrire dans un va-et-vient constant entre l'Europe classique et le(s) pays du Soleil levant. Entendons-le, dès les toutes premières lignes du texte, se livrer aisément à l'objet instrumental qui l'accompagne :

Et qui m'empêcherait, Monsieur Peau de Chat, de calligraphier $[\ldots]$ une $[. .$.$] relation de mon voyage, station$ par station, dans le goût de ces anciens itinéraires japonais $[. .$.$] [des] chemin[s] peint[s] en jaune à travers [...]$ de[s] bosses vertes] comme on voit sur ces grands tableaux au sortir des gares ${ }^{2}[\ldots]$ ?

De prime abord, l'objectif du poète semble défini : l'emprunt à la calligraphie, à cet art de l'idéogramme, comme intention première, laisse entrevoir une mixité d'allusions à l'Orient et à ses symboles, englobant la figure du tigre, et le paysage inhérent à la peinture asiatique. Puis, s'enchevêtrent la religion, la sienne, à travers La Relation des Jésuites, et le Chemin de croix du chrétien, entre autres, selon ma propre lecture ${ }^{3}$.

Tous ces ingrédients participent d'un échange avec l'autre.

Claudel semble amusé d'entreprendre cet itinéraire. Il présente ce divertissement sous l'influence d'une genèse théâtrale 


\section{Hélène Poiré}

soumise à l'apostrophe et à la transcription d'un message. Le tout engrangé avec subtilité, laissant poindre, néanmoins, les accents insoupçonnés d'une âme sans cesse inquiète de son devenir intérieur.

Ainsi, dans un face-à-face avec le shamisen, ou l'intervenant salutaire, le poète s'agite au rythme d'accords impromptus, facilitateurs de sa démarche.

Bref, l'instrument demeure un quelconque garde à vous indispensable.

Narrer certains faits et gestes du voyageur au sein de cette mélopée inscrite au cœur de l'Orient, permettra, je le souhaite, de débroussailler ses comptes rendus des mondes vrais ou imaginaires, peuplant sa rencontre avec de multiples cultures.

Claudel, l'homme d'hier, à découvrir, au carrefour du temps d'aujourd'hui.

\section{Motivation intrinsèque de l'auteur}

Saisir, à rebours, la motivation de cet émissaire français, né avec le goût de l'autre, l'instinct de la découverte, et nanti de ce don irrécusable pour les mots, est devenue l'achoppement à vaincre.

D'où le désir de raconter le plus de cet être d'exception.

Quelles intentions avaient en tête le poète, même inconsciemment, au cours des années 1923 à 19264, au moment particulier de l'écriture de cette nouvelle, laquelle s'avère, au premier regard, un simple dialogue du poète avec lui-même ? Claudel $a-t$-il voulu ficeler les impressions vécues, laisser errer son imagination sur les paysages et les éléments culturels auxquels il fut partie liante ou, simplement, "se dégourdir les doigts », puisque, du même souffle, il était alors sur place pour travailler à des fonctions diplomatiques, et qu'il besognait depuis cinq ans, soit, de 1919 à 1924, sur l'écriture plus solennelle du ... Soulier de satin, dans la poursuite de son œuvre dramatique ${ }^{5}$ ?

À cette époque, fort heureusement pour nous, l'ère de la technologie scripturale n'étant pas née, les penseurs du temps, pressés par la réflexion et l'analyse, étaient aptes à créer des lignes 
profondément circonscrites, et ce, de façon perfectible. Les défis étaient à la mesure du talent entrepreneur. De fait, Claudel quittera le Japon, quelque temps à peine après cette écriture, d'où émanent la redondance des sites qui se déroulent à la façon des rouleaux japonais, et les vibrations sensibles suite à la vision de spectacles, tels le Nô et le Bunraku, en particulier. Spectacles que le poète s'emploie à décrire, avec force détails, au seuil de l'entreprise de ce recueil, susceptible d'être encore qualifié de Journal à tenir. Échos de pages antécédentes - premières - dans lesquelles le poète résume ainsi sa version du Nô : « [Q]uelqu'un qui arrive » sur une scène composée d'un Chemin qui sert de Pont à l'Estrade, $a$ contrario du drame, insiste-t-il,... qui est « quelque chose qui arrive ». Ici, le spectacle et le spectateur « entrent l'un dans l'autre ». Le déploiement des acteurs sur deux plans latéraux permet au spectateur, depuis son siège, de se créer « une géométrie personnelle », en lien direct avec l'angle virtuel formé par son œil et son oreille. Aussi ranime-t-il l'art de la Marionnette japonaise, domestiquée au cours des ans, pour atteindre à la suprématie du Bunraku : « [E]lle s'anime sous le récit [cette marionnette] comme une ombre qu'on ressuscite [...]. [C]'est une parole qui agit ${ }^{6} »$.

Ce tout, si intimement lié, à décloisonner, depuis la vie vécue, et celle ressentie par l'auteur; cette narration sans cesse interrompue par la cadence des accords du shamisen, ce luth à trois cordes - rappel de l'acteur infatigable du Bunraku, pouvant être dit, aussi, celui de la perpétuité d'un peuple - Claudel accueillant cette respiration pour mieux démythifier ses passions, son dynamisme culturel, et surtout, apaiser sa rencontre avec une religion autre, le bouddhisme.

Ainsi va le poète à la recherche de lui-même tout au long de cet itinéraire.

\section{Les sources modélisantes}

Tel le pèlerin de Compostelle, le compagnon Claudel renoue avec ses racines intérieures. Rien ne lui échappe. De l'im- 


\section{Hélène Poiré}

pression outrancière, venue de Valéry et du poème Îles, jamais intitulé comme tel par ce dernier, aux paroles arrachées plutôt, à [Sa] Jeune Parque... mimesis fortement empruntée au poète ami, dont il explicite la formule avant de le citer dans un verset, ou deux : « Je lisais, écrit Claudel, ce fragment séparé par des lacunes du reste de la Jeune Parque qui par son isolement et sa construction superposée donnait [...] une impression [...] d'île et de montagne ». Et surgissent les mots de Valéry : «Salut! divinités par la rose et le sel, [...] Iles! [...] Iles! [... $]^{7}$ ». Ambiguïtés à dénouer. De fait, " [Iles] un mot [irradiant] toute l'œuvre de Valéry », selon Pierre Caminade. Ce dernier ajoute que le poète inclut, à coup sûr, l'Ile intérieure, [soit], une part de sa vie intime, empreinte à la fois de " solitude [et] d'ouverture, [...] [de] retraite [...] tremplin [...] [et de] forteresse rebelle $[\ldots]^{8}$ ". D'où l'éclatement de son origine. Étant né d'un père corse, l'île a bien eu lieu.

$\mathrm{Si}$ aspiré par la pensée valéryenne, son contemporain, on admet que Claudel se soit substitué à un symbole aussi prégnant, lui qui séjourne au Japon... De là l'émergence du grand fleuve bleu, le Yang-Tsé, où se terre un amas d'îlots; ce fleuve qui isole du continent asiatique, métonymie de la montagne, d'où le regard obligé, de bas en haut, s'incarne pour simuler la verticalité.

Plus près de lui, à ce moment-là, Claudel éclairé par la pensée de Lao-Tzeu revisite Hirogishé, ses paysages colorés, ses murmures transcendants, toujours hanté par l'exaltation envahissante devant une nature à laquelle il prête la qualité du surnaturel $^{9}$. De bas en haut se liront presque toujours les toiles de l'Orient - vertu ascendante - qui porte les yeux vers les sommets pour se confondre avec l'immobilité, le silence... Extase provoquée par la réunion du ciel et de la terre, afin de définir la perfection du paysage $^{10}$.

Claudel s'est donc mis en marche pour célébrer « $[. .$.$] ce$ [que le spectacle] veut dire [surtout] ${ }^{11} »$, à l'instar de Mallarmé, duquel il a usurpé la réponse. Car il a été déterminé, plus avant, qu'il s'agit bien, ici, d'une mise en scène... Le réalisateur Claudel a retenu la leçon, il la récitera, en ces termes, plusieurs années plus tard : 
Je ne me suis jamais placé devant un spectacle avec la sensation de m'y perdre, mais au contraire de le dominer et de tâcher d'en réunir les éléments ${ }^{12}$.

\section{La direction prend divers sens}

Et si « l'île » était devenue spectacle, ne pourrait-elle aussi signifier le « il », pronom, le soi représentatif d'un ego insistant, en voie de se raconter les lieux et les souvenirs enfouis, dans les limites de sa propre interprétation! De fait, Claudel accentue « [1]'acharnement pédestre [et sa] fidélité rectiligne à [son] propos », alors que Shamisen réplique que cette «fidélité [du poète » le reporte] au véritable bunraku, [vu à] Osaka, deux ans [plus tôt] ". Produit imaginatif s'il en est, créé par le poète, selon Shamisen. Il s'éloigne de la description originale en comparant les marionnettistes à de simples animateurs, chacun recréant sous l'effet de l'ombre, soit, « un corps [embrasé par un faisceau] lumineux », ou d'autres ombres semblables « à [des] enveloppes vides de soi [...], [et même encore] à [un] buisson d'apparences confuses, [...] sans cesse [...] réintégré ou déserté [par] tout être vivant $»^{13}$. Au fond, le poète Claudel fustige un passé proche lui intimant sa version de l'événement. Bien plus, il y imprime un symbole, quasi biblique, avec le concept «buisson » que l'on a tendance a qualifié d'ardent. L'obsession du corps est palpable. D'où le feu sacré qui en émane. Ardeur sans cesse renouvelée... Sa vision, agie sur le plateau, fait naître des marionnettes à plusieurs têtes, partant, plusieurs tableaux.

Le poète a-t-il voulu modifier les règles du Bunraku originel, imprimer sa marque, ou infléchir le comédien? Lui-même, dubitatif, hésite encore à définir lequel de ces tableaux serait à retenir pour en divulguer les fondements, à des fins de rappel, ou de continuité... C'est avec effervescence, néanmoins, qu'il poursuit son tempo. S'il propose aussi un « il » double, à la fois instrument et personnage, il laisse libre cours au spectateur/lecteur de décider du choix du réel intermédiaire. Un scepticisme certain s'installe, 


\section{Hélène Poiré}

quant à la logique de cette pensée, jugée plutôt vagabonde, ici, balisée par les seuls contours du paysage au sein duquel le comédien Claudel se meut... Il escalade sa montagne tout en s'arrêtant à plusieurs haltes, afin de s'élever sur ses hauteurs pour mieux contempler le paysage. Sisyphe n'est pas très loin... et veille au bien-être de son préposé, lui épargnant de rudes contraintes, multipliant le chemin parcouru en autant d'autres voies possibles... Le poète semble arpenter une toile de Riopelle; il entre et sort s'imaginant devant « un grand panneau carré » où il pourrait se livrer à des entrées et des sorties de plusieurs côtés à la fois, ainsi poursuivrait-il " une espèce de centre jamais atteint ${ }^{14}$ ».

L'excitation visuelle est à son comble. Point de supercherie, Claudel, l'artiste devenu, se déploie, adoptant un ensemble de coloris perceptibles dans les allusions à divers lieux naturels décrits. Puis, modulant quelque peu sa pensée, il succombera « au modelé » des toiles vénitiennes, d'une facture autre, d'une époque autre, desservant le Corrège et le Titien dans l'infini d'une sensualité appréhendée, dont la Vénus d'Urbin, 1538, de ce dernier, peutêtre... qu'il s'abstiendra même de nommer. Il sous-entendra encore La Grande Odalisque d'Ingres ${ }^{15}$.

Nonobstant quelques répétitions dans les thèmes abordés, on s'interroge à savoir pourquoi, un Claudel rationaliste admet chercher une direction? Il importe de se tourner, une fois de plus, du côté des interrogations scientifiques de Valéry pour en extirper la substance ${ }^{16}$. Ce guide, tant prisé par Claudel, un double insoupçonné à certains égards... comme si sa propre plume devait reposer sur la dictée d'un maître, alors qu'il est engagé sur le chemin horizontal, dégagé en cet instant des considérations géométriques valéryennes...

Peu s'en faut qu'il ne la trouve à l'intérieur de lui cette direction, si ce n'est dans la conduite d'une vie sans cesse en recherche de la complétude la plus parfaite!

Claudel aurait-il désespéré, à cette époque, de sa propre humanité ? Peut-être... il a trop vu, trop lu, trop entendu, trop 
échangé, trop écrit, trop aimé, pour se dire à l'abri des contingences auxquelles sont soumis ses semblables.

Du reste, on est de plus en plus mis en présence d'un personnage qui retient en lui les mobiles mêmes de ce moment interrogatif.

\section{L'architecture trouble de plusieurs mondes}

Deux entités majeures semblent conduire Claudel à l'apogée du questionnement : d'une part, le théâtre de marionnettes qui, tantôt le fascine, à travers les données japonaises, d'autre part, l'image millénaire, Lao-Tzeu, qui ramène tout critique à la peinture du paysage chinois, là où l'on devrait trouver « la solution à tous les problèmes ". Le tout animé par la poésie, jugée très terre à terre par Claudel : «Quelque chose d'inspiré, [certes, écrira-t-il... mais], de noir, de délabré, de vide $[\ldots]^{17}$ ». Cette définition claudélienne de la poésie étonne... l'auteur paraît vraisemblablement confronté à un statut professionnel ambivalent, lorsqu'il écrit ces lignes. Il est, à la fois, à l'intérieur et à l'extérieur de lui; il se joue son propre drame, accentué par l'univers dans lequel il est inscrit, en écho peut-être... aux personnages $\mathrm{du}$...Soulier de satin dont il vient, à peine, d'achever les rôles ${ }^{18}$. Claudel, à la fin de sa vie, fustigé par Jean Amrouche qui prétend que ce drame constitue « un argument philosophique » majeur, se laissera relire un fragment de l'une de ses lettres de 1909, à cet effet. Le dramaturge écrivait, alors, à propos de sa pièce, qu'il s'agissait de « [...] la lutte de l'invisible contre le visible [...], [la scène étant le lieu de la recréation] [d'un] monde géographique, [d'un] univers planétaire [...] et aussi [d'un] monde surnature ${ }^{19}{ }^{\prime}$; sorte de décloisonnement des mondes claudéliens.

D'où sa déception, vis-à-vis la direction rectiligne entreprise, à l'époque de L'oiseau noir...comme si son propre conformisme religieux le maintenait dans une seule voie, l'empêchant de capter les différences religieuses de l'autre, alors qu'il endosse un contemporain, nommé Grantham, dans son affirmation 


\section{Hélène Poiré}

liée aux « lois de la composition [qui découleraient] du sens [même] de [cette] direction » imputée au gabarit et à l'éventail des objets peints sur la surface choisie ${ }^{20}$.

Afin de mieux écarter sa propre dissonance au sein de ce milieu tant admiré, Claudel bascule, substituant à la puissance d'un peintre japonais, Wang Wei de l'époque des Tang, louangé par l'auteur précité, l'exploitation « d'une espèce de bassin d'idées ${ }^{21}$ », germe d'interrogations multiples, rejoignant en ceci, « les génies du point d'interrogation ", les Japonais eux-mêmes, tels que décrits par André Malraux ${ }^{22}$. L'intérêt, hors du commun, exercé par ce pays, sur Claudel, y compris ses habitants, ses us et coutumes, risquait de lui faire perdre la tête et oublier ses engagements.

Cette conjoncture peut expliquer son emprunt à la vocation du peintre, pour un instant... En bref, le libéralisme de l'artiste peintre, éloigné des mots, traitant la nature avec sérénité et un contact visuel permanent, l'absout de toutes fautes, y compris celle de se détourner de la religion bouddhiste, à laquelle il admet ne pas appartenir. Paradoxe... puisque le bouddhisme s'apparente à la transmission de l'image. On croit entendre Mircea Eliade lui souffler à l'oreille de se nourrir d'images vues et contemplées, qu'elles soient réelles ou imaginaires :

\section{[...] [L]'homme le plus « réaliste » vit d'images. Pour le redire, [...] les symboles ne disparaissent jamais de l'ac- tualité psychique : ils peuvent changer d'aspect; leur fonction reste la même. Il n'est que de lever leurs nou- veaux masques ${ }^{23}$.}

Un visage autre à emprunter pour Claudel? Cette religion était peut-être celle dont il rêvait le plus, puisqu'elle réunissait le tout de l'homme : ses aspirations mortelles face à la contemplation d'une nature vraie, celle à laquelle il était très attaché comme pèlerin du monde, outre son incessante poursuite de la vérité et son désir, inconscient, d'amener les âmes à son Dieu.

On doit tout de même constater que tout ce qu'il voit le ramène à un monde connu, d'où la certitude d'un aller-retour entre 
divers mondes, et celle d'un tour de l'histoire du monde initié, on l'a vu, par La Jeune Parque, demeurée un souvenir vivant... ce poème de Valéry dont il a fait l'éloge.

La fréquentation continue de ces « ombres doubles » issues, sans contredit, du ...Soulier de satin, a pris si fortement ancrage que l'on a tendance à s'identifier à elles pour mieux parachever le discours. Aussi est-il pertinent de renouer avec les deux personnages dramatiques principaux de l'œuvre précitée de Claudel, soit, Don Rodrigue et Doña Prouhèze... cette dernière en mal d'amour, pour y percevoir une allusion dévolue aux seuls personnages du Nô : Waki, « celui qui attend », et l'autre, Shité, « l'Ambassadeur de l'Inconnu ", révélé sous le masque, face aux spectateurs, sous le prétexte d'être "l'acteur de sa propre pensée » [... ${ }^{24}$. L'idée d'un transfert des dits personnages au Poète et au Shamisen est renforcée... ce dialogue ayant pu tirer son origine, et de l'œuvre dramatique, et de la connaissance du Nô... un reflet du monde. En définitive, les deux phénomènes simultanés concourent à raffermir la superposition possible de divers mondes, sous-jacents à la pensée même de Claudel. Si le dramaturge se fait fureteur, c'est que tous les mondes l'habitent, y compris son allant vers l'autre qui l'emprisonne dans le milieu présent du texte.

\section{Aux confins de l'effritement du corps-sujet}

Sa difficulté à saisir l'âme, l'intériorité de ce peuple, indique peut-être la raison pour laquelle Claudel se soit rallié à la fantasmagorie pour l'énoncer. La marionnette dissimulée sous le masque lui facilite mille facéties. L'exploration de cette route qui n'en finit plus... lui permet aussi d'extrapoler sur sa vision des paysages, qu'il tente de conquérir par sa lecture personnelle.

Puis sursaut... au beau mitan de cet itinéraire, le poète est ébranlé par l'hara-kiri - l'éventrement du corps - naissance de la blessure rouge qui lui fera confondre, tel un imbroglio, «la rage de s'enfuir, de sortir de l'île », au plein séjour ${ }^{25}$. Une lecture ponctuelle m'habite, ici... « l'héritage [anthropologique] de Gilbert Durand » 


\section{Hélène Poiré}

est toujours d'actualité. Dans son interrogation sur « l'être au monde [...] devant le temps », il a entraîné à sa suite, Jean Burgos. Ce dernier s'est lancé dans l'analyse d'une syntaxe de l'imaginaire, proposant trois types d'écriture poétique. Les schèmes qui en ont découlé, soit, la révolte, le refus, ou la ruse, m’ont semblé révélateurs de l'une ou l'autre attitude possible, chez le poète, compte tenu de sa manière d'être, ambiguë, à ce moment précis ${ }^{26}$.

Mais, avant de quitter son Île, dans une volte-face, il déambule, à nouveau, sur le chemin nippon. C'est le mouvement qui l'habite, la vie autour de lui, recelée dans les fleurs, les bouquets, « un [...] jardin bouddhiste [particulier, celui] de Hasédéra », et le monde vivant, tout à côté... Le poète et le luth chantent à l'unisson, mais départagent le fruit de leurs émotions. Si toute beauté renferme un élément de tristesse, chez l'un, l'autre, le poète, ressent " l'amère paix du paradis bouddhiste [quasi comme un] enfer ". Dichotomie certaine. Le Nô intervient, prête son ambiance, composée à la fois de «mystère et de terreur ${ }^{27}$ ». Même si Claudel, à travers des images prégnantes, veut créer de l'enchantement alentour des choses vues : la nature et l'art, l'ambivalence des sentiments demeure... Son désir parallèle, quasi obsessionnel, de s'accrocher à la géométrie, de s'en imprégner comme ligne de conduite immanente, refait surface tout au long de ce parcours. La direction de la ligne engagée pose toujours problème...

Le poète, en dernier ressort, face à la Pagode, à caractères géométriques, s'immobilise pour contempler le triangle aux angles arrondis, rappel du fronton grec... mais la négation des arêtes vives engendre aussitôt le pouvoir de la courbe.

L'assertion suivante de l'auteur livrerait-elle le paradigmeclé, sur lequel devrait s'appuyer le lecteur, pour définir l'essence même de ce message claudélien? De fait, en conclusion, ou presque, il confie le relais à Shamisen. Il lui met en bouche un propos radical :

Il n'est pas nécessaire « d'être bouddhiste pour croire [...]

à une porosité à certaines influences [...] il y a en nous 
[...] la succion de notre temps personnel par l'immense temps de la nature ${ }^{28} »$.

Claudel préfère se glisser dans la peau du luth, pour apporter un démenti à la nécessité d'une culture religieuse particulière pour avoir le droit d'accéder à l'ensemble des cultures. Lutte, sans trêve, oserai-je écrire... infiniment capitale, pour cet être demeuré, jusqu'à la fin de ses jours, un être de raison!

\section{Au terme de ce voyage...}

Agglutinée, moi-même, à la parole des deux intervenants, la question première posée, loin d'être obsolète, ressurgit avec plus d'impératifs. Itinéraire pédestre sans précédent... retour quelconque sur lui-même, Claudel construit son aventure comme une mise en scène au cour de laquelle il fait surgir des paysages à la façon de toiles d'artistes orientaux, surtout, dans lesquelles il insère un lot de représentations et d'événements artistiques vécus, et d'autres, étendus sur plusieurs siècles. Est-ce ainsi, toutefois, que le poète aurait aimé être discuté ? N'a-t-il pas voulu, plutôt, livrer son texte à la façon d'idéogrammes, additionnant les signes, emmagasinant le maximum de données, et même celles du milieu occidental, avec en tête la quasi certitude que ce procédé rendrait justice à sa reconnaissance des cultures, très conscient de l'interpénétration de toute culture sur une autre.

L'ascension légendaire qui le conduit indubitablement à refaire un parcours visuel artistique, toutefois, ne peut être niée... comme si la condensation d'un rappel historique - personnel allait lui conférer plus de crédit dans ses descriptions arbitraires des lieux exploités, ou mieux encore, allait lui faciliter la rupture présente d'avec cet Orient extrême, en vue d'un retour vers l'Occident suprême, l'Amérique ${ }^{29}$. Il faut convenir, toutefois, qu'une aspiration certaine du lieu est établie, en raison d'un appel majeur, soit, une autre fonction diplomatique à débuter, et déjà résolue dans une respiration adaptée, avec lui-même... 


\section{Hélène Poiré}

Lorsque le rideau tombe et que les marionnettistes se sont retirés, emportant avec eux les vestiges de ces personnages, désormais démembrés... Claudel, en confidence, se livre une toute dernière fois au luth... à l'instrument musical, quasi charnel, à l'habile serviteur de ses dires, lui dévoilant le but précis de son projet immédiat, sa vision de l'Amérique, par le biais du Gouverneur de La Caroline $d u$ nord, et du sud, profession oblige...

J'ai le goût, ici, de suggérer un dernier double, à la fois " tendre et terrifiant » à venir, cette Agnès Meyer, qui l'attend outre-Atlantique, à arracher du mal. L'auteur se complaît dans le symbolisme, comme s'il s'agissait d'une autre peau à laquelle il se devait de s'ajuster.

En résumé, Claudel semble s'être plu, à travers l'ouïe, à fidéliser un contact possible en attente... une liaison inespérée! La voix épistolaire, tout au moins, présente un alibi parfait. Lisons-le plutôt :

La rencontre de l'autre [quelle que soit la forme empruntée], sert de prétexte au déploiement de visions. L'Amérique est la terre de la démonstration et de l'évidence. [...]. Elle est aussi le pays des secrets et des mystères ${ }^{30}$.

La forme italique des derniers mots, utilisée par Claudel, est une insinuation sentie dans cette lettre adressée à Agnès Meyer, peu de temps avant son départ du Japon. Cette voix de chair, inconsciente, à travers les accents muets, risquait de meubler la distance - de reculer le moment de sa rencontre - refoulant son désir, lequel à l'époque pouvait ressembler davantage à un engagement mythico religieux, qu'à la saisie d'une femme dont il se défendait...

\section{Notes}

1 Bernard Hue, Littératures et arts de l'Orient dans l'Euvre de Claudel, Paris : Klincksieck, 1978, p. 380. 
2 Paul Claudel, L'oiseau noir dans le Soleil Levant, NRF, Paris : Gallimard, 1929, p. 149.

3 On ne peut guère s'éloigner du « religieux », lorsque l'on fréquente Paul Claudel. Aussi importe-t-il de souligner le " coup de grâce » reçu, en ce 25 décembre 1886, à l'heure des Vêpres, à Notre-Dame, rapporté par Jean Amrouche, et confirmé par Claudel, lui-même. Cet instant installait le poète, dans un élan, vers la concrétisation de son appartenance à la foi chrétienne. Paul Claudel, Mémoires improvisés recueillis par Jean Amrouche, Les Cahiers de la NRF, Sixième entretien, Paris : Gallimard, 2001, p. 55.

4 L'identification des textes très inégaux, de ce recueil, me fait osciller entre quelques choix : chroniques, nouvelles, journal de bord, même... Ce qu'il importe de retenir, c'est leur authentification dans le temps. Le tout premier de ces textes aurait été écrit en 1923, et le dernier, en 1926.

5 Claudel avouera à Amrouche que « des considérations personnelles », qu'il veut taire, à ce moment-là, sont parties liantes des éléments abordés dans Le Soulier de satin., Trente-quatrième entretien, 2001, p. 279.

6 Paul Claudel, L'oiseau noir dans le Soleil Levant, op. cit., p. 86-90. L'histoire du théâtre japonais, aussi nommé théâtre d'ombres, serait à développer pour une meilleure compréhension des dires mêmes de Claudel qui s'en est inspiré, à coup sûr... Un ouvrage récent, capital, sur le sujet, œuvre de Fan Pen Li Chen, est à consulter : Chinese Shadow Theatre, History, Popular Religion, and Women Warriors. Montreal \& Kingston, London - Ithaca : McGill-Queen's University Press, 2007. À lire, entre autres, le chapitre I, Introduction, p. 3-13, et les notes accompagnatrices, p. 235.

7 Paul Claudel, L'oiseau noir dans le Soleil Levant, op. cit., p. 157-158, et Paul Valéry, Morceaux choisis : prose et poésie. "La Jeune Parque ». Paris : Gallimard, 1950, (cl 1930), p. 20.

8 Pierre Caminade, Valéry, Vérones : Pierre Charron et Arnoldo Mondaderi, 1972, Chapitre, "Valéry intime », et sous-titre, "L'Île intérieure », p. 26.

9 Qu'il suffise de se pencher sur la recherche de Hubert Delahaye, pour se convaincre d'explorer plus avant la notion même du «surnaturel ». De fait, l'auteur, exégète de la langue chinoise, apporte en définitive - la réponse bouddhiste - à la représentation visuelle de la peinture, soit, qu'elle conquiert "l'esprit - cosmique - dans la forme des choses ». Une recherche ultérieure s'imposerait pour déceler les 


\section{Hélène Poiré}

composantes de la dimension spirituelle de "la peinture chinoise" perçues, par Claudel athée, devenu chrétien. In Les premières peintures de paysage en Chine : aspects religieux, Paris : Publications de l'École française d'Extrême-Orient, volume CXX1X, 1981, p. 102-105

10 Patrick Riggenberg, L'union du ciel et de la terre : la peinture de paysage en Chine et au Japon, Paris : Collection Les Deux Océans, 2004, p. 122-123. Voir aussi les pages 16-17, en particulier.

11 Paul Claudel, Mémoires improvisés, op. cit., Dix-septième entretien, p. 145.

12 Ibid, p. 149.

13 Paul Claudel, L'oiseau noir dans le Soleil Levant, op. cit., p. 151.

14 lbid, p. 152-153.

15 Ibid, p. 159 et p. 164. On assiste, dans ces pages, à un délire de mots et d'images composites, (un quelconque papier collé), qui créent, ou recréent un paysage claudélien, tracé de souvenirs européens issus de la fréquentation des musées de chez-lui., et de la mémoire certaine de sa célèbre sœur, l'artiste Camille.

16 Une formule valéryenne, parmi tant d'autres, à retenir. Pour Valéry, " le langage [de l'esprit] des mathématiques [...] fut plus qu'un beau projet $[. .$.$] il a essayé de l'ébaucher lui-même [...]». Judith Robinson,$ L'analyse de l'esprit dans les Cahiers de Valéry, Chapitre 111, "Vers une mathématique de l'esprit", Paris : Librairie José Corti, 1963, p. 62.

17 Paul Claudel, L'oiseau noir dans le Soleil Levant, op. cit., p. 152.

18 L'assistant du metteur en scène du Soulier de satin raconte que cette histoire d'amour reflète « le combustible de toute une vie, [...] transfigurée par la poésie. [...] [Une] vocation à l'univers [confessée], de l'Afrique à l'Orénoque, de Prague à Panama [...]». Il ajoute encore : "Jouer Le Soulier de satin, écouter, c'est faire un voyage. On passe d'un continent à l'autre, sans transition, et [...] sans bruit ». L'auteur de ces dires a participé à la première présentation de cette œuvre. Éloi Recoing, Journal de bord, Le Soulier de satin de Paul Claudel [Antoine Vitez, metteur en scène], Paris : Éditions Le Monde, 1991, p. 25.

19 Paul Claudel, Mémoires improvisés, op. cit., Trente-quatrième entretien, p. 283.

20 Paul Claudel, L'oiseau noir dans le Soleil Levant, op. cit., p. 155.

$21 \mathrm{Ibid}$, p. 156.

22 André Malraux, L'Intemporel, La métamorphose des dieux, NRF, Paris : Gallimard, 1976, p. 229. Les chapitres 7 et 8, entre autres, regorgent 
du sentiment chinois et japonais, et des acquis nombreux transmis par ces peuples, en regard de leur art millénaire, p. 169-241.

23 Mircea Eliade, Images et Symboles. Essais sur le symbolisme magico religieux, Paris: Gallimard, 1952, p. 19.

24 Paul Claudel, L'oiseau noir dans le Soleil Levant, op. cit., p. 93-94 et p. 96.

25 Ibid, p. 160-165.

26 Jean Burgos, "La Poétique de l'imaginaire" p. 77-85, in L'Imaginaire littéraire, Des archétypes à la poétique du sujet, sous la direction de Henri Mitterand, (cl. Paris : 2000), et Paris : Armand Colin, 2005, p. 80-81.

27 Paul Claudel, L'oiseau noir dans le Soleil Levant, op. cit., p. 166-167.

28 Paul Claudel, L'oiseau noir dans le Soleil Levant, op. cit., p. 170.

29 Claudel avait séjourné, brièvement, à New York, en 1893, à titre de consul suppléant, ainsi qu'à Boston, en 1894, comme gérant du Consulat. Lire «À propos de la correspondance Claudel-Pottecher », par Pierre Moreau, avant que le diplomate quitte pour Shangaï, en 1895. On y apprend, entre autres, dans une lettre du 17 janvier 1894, adressée à son ami Maurice Pottecher, vivant à Paris, que « [Claudel regrettera] l'Amérique et ces ciels d'une pureté exquise ! ». In Cahiers Paul Claudel, vol. 1, NRF, Paris : Éditions Gallimard, 1959, pp. 62 et 88.

30 Claudel et l'Amérique 11. Lettres de Paul Claudel à Agnès Meyer, 1928-1929, Notebook d'Agnès Meyer, 1929, Éd. établie avec intr. et notes par Eugène Roberto, Canada: Université d'Ottawa, 1969, p. 16. 